Article

\title{
A New Alkylation of Aryl Alcohols by Boron Trifluoride Etherate
}

\author{
Ndze Denis Jumbam, Yamkela Maganga, Wayiza Masamba * $\mathbb{D}$, Nomthandazo I. Mbunye, \\ Esethu Mgoqi and Sphumusa Mtwa \\ Department of Chemical and Physical Sciences, Faculty of Natural Sciences, Walter Sisulu University, Nelson \\ Mandela Drive, Mthatha 5117, South Africa; njumbam@wsu.ac.za (N.D.J.); magangayamkela@gmail.com (Y.M.); \\ mbunyenomthandazo@gmail.com (N.I.M.); esemgoqi12@gmail.com (E.M.); mtwasphumusa@gmail.com (S.M.) \\ * Correspondence: wmasamba@wsu.ac.za
}

Academic Editor: Narciso M. Garrido Received: 10 September 2019; Accepted: 9 October 2019; Published: 16 October 2019

\begin{abstract}
The ethylation of aryl alcohols by an ethyl moiety of boron trifluoride etherate is described. The reaction proceeded cleanly and afforded good yields of the corresponding aryl ethyl ethers. It tolerated the presence of functional groups such as aryl, alkyl, halogens, nitro, nitrile, and amino. However, the presence of amino or nitro groups ortho to a hydroxyl group of an aryl compound drastically reduced the yields of the anticipated products due to the chelation of the aforementioned functional groups with boron trifluoride etherate. A nitrogen atom in the aromatic ring system, as exemplified by hydroxypyridine and 8-hydroxyquinoline, completely inhibited the reaction. Resorcinol, hydroquinone, and aryl alcohols with aldehyde functions decomposed under the reaction conditions.
\end{abstract}

Keywords: aryl alcohols; aryl ethyl ethers; boron trifluoride etherate; ethylation; functional groups

\section{Introduction}

The unprecedented ethylation reaction of carboxylic acids by an ethyl moiety of boron trifluoride etherate (BTE) to form corresponding ethyl esters in the absence of ethyl alcohol may have opened up a new synthetic route for the formation of aryl ethyl ethers, thanks to the observed simultaneous formation of ethyl $p$-ethoxybenzoate from $p$-hydroxybenzoic acid [1].

Aryl ethyl ethers find varied applications in industry ranging from solvents, paints, cosmetics, and pharmaceuticals [2]. For example, $p$-ethoxyaniline is the precursor for the production of the analgesic phenacetin [3,4], $\beta$-ethoxynaphthalene (also known as nerolin) is used as a fixative in perfumery [5] while some derivatives of aryl ethers exhibit antioxidative properties [6]. In light of the aforementioned properties of aryl ethyl ethers and their potential applications in the chemical and pharmaceutical industries, we set out to explore the alkylation of various phenols using this new ethylating agent.

The traditional Williamson ether synthesis, outlined in Figure 1, involves the $\mathrm{S}_{\mathrm{N}} 2$ reaction of an aryloxide with a primary alkyl halide, or of an $\mathrm{E}_{\mathrm{AR}} \mathrm{S}$ reaction of an alkoxide with an aryl halide [7], in the presence [8,9] or absence of a solvent [10].

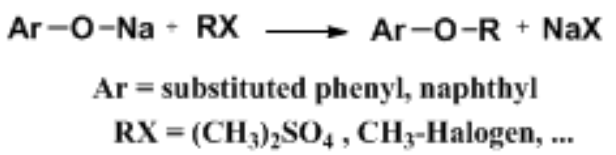

Figure 1. Conventional Williamson ether synthesis of alkyl aryl ethers.

Though effective and generally considered to be the method of choice, the Williamson ether synthesis suffers from some drawbacks such as the toxicity of the associated alkyl halides and 
dimethyl and diethyl sulfate. In addition, some alkyl halides involved in this reaction are prone to elimination under basic reaction conditions. The $\mathrm{Cu}$-promoted Ullmann ether synthesis [11] and its variants $[8,12,13]$ are a useful alternative method, but is limited to certain reactive substrates and only takes place in high boiling polar solvents. The Mitsunobu protocol, whereby the relevant phenol and alcohol are condensed in the presence of diethyl azodicarboxylate and triphenylphosphine, smoothly allows access to the expected alkyl aryl ethers in good to excellent yields [14]. However the resultant triphenylphosphine oxide poses tremendous challenges during product purification. Recently, an alternative version of the Williamson ether synthesis using dialkyl carbonates and imidazole carbamates as the alkylating agents [4,15-17] has been proposed. Because BTE is mostly used as catalyst at low temperatures, its capacity to act as an alkylating agent has eluded the attention of organic chemists until now. We wish to report here our findings on its high temperature reaction with variously substituted phenols.

\section{Results and Discussion}

The results obtained in the reaction of various aryl alcohols with boron trifluoride etherate (BTE) are summarized in Figure 2 below.

Various aryl alcohols were ethylated under the above reaction conditions affording the corresponding ethers in low to excellent yield depending on the substitution on the aromatic ring. The reaction tolerated certain substituents such as, alkyl (compounds 7-9), halogens (compounds 2, 3, 5 , and 6), nitro (compound 4), nitrile (compound 13) and amino (compound 10). The influence of some substituents, such amino, hydroxyl and nitro, on the overall outcome of the reaction depended on its nature and its position relative to the reaction center. Thus, while $p$-nitrophenol was converted to p-ethoxynitrobenzene (compound 4), its ortho isomer (o-nitrophenol) failed to react under the same reaction conditions, most likely due to the steric hindrance brought about by the proximity of the nitro to the hydroxyl group. The amino group on the other hand showed a somewhat different trend: $p$-aminophenol was ethylated, albeit in low yield (compound 10) after refluxing overnight. Its ortho isomer (o-aminophenol), on the other hand, formed the corresponding ether only in trace amounts and the slow reaction could also be attributed to the steric hindrance between the two adjacent groups (amino and hydroxyl). All three isomeric hydroxyphenols led to decomposition products, forming tarry materials. A nitrogen atom forming part of the aromatic ring system completely inhibited the ethylation reaction as exemplified by $p$-hydroxypyridine and 8-hydroxyquinoline, probably due to the chelation of the nitrogen atom onto the Lewis acid, leading to the formation of resinous materials. The presence of a nitrile group slowed down the ethylation reaction considerably (compound 13) and only $20 \%$ of the anticipated product was obtained after refluxing the reaction overnight. As in all the cases where low yields are obtained, the starting material was not recovered and was completely converted into tars. All three isomers of hydroxybenzaldehyde decomposed and formed resinous materials. The decomposition of $p$-hydroxybenzyl alcohol, on the other hand, may not be surprising given that hydroxybenzyl alcohols are prone to polymerization under acidic conditions [18].

BTE promotes the formation of a carbocation with aliphatic hydroxyl groups, which subsequently undergo alkene formation if $\beta$-hydrogen atoms are present [19]. The alkenes formed in the process are likely to polymerize under the above reaction condition as observed in our previous investigations wherein undecylenic acid was esterified to ethyl undecylenate followed by the polymerization of the double bond [1]. Although the alkylation of carboxylic acids and ethylation of phenols were carried out in BTE as solvent, preliminary experiments done using this reagent in stoichiometric amounts without a solvent have proved to be equally successful. In the presence of solvents such as toluene and 1,2-dichloroethane the reactions were too slow to be of any significant value. The mechanism of the ethylation reaction is believed to involve the coordination of the oxygen atom of the hydroxyl group with BTE, followed by the transfer of the ethyl group by alkylation as depicted in Scheme 1. 

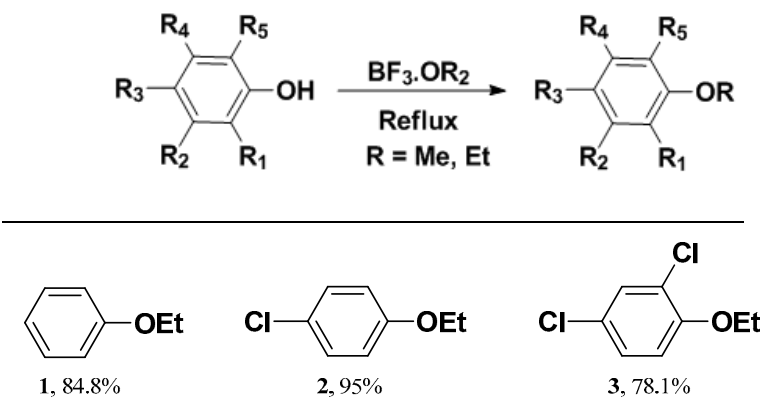<smiles>CCOc1ccc([N+](=O)[O-])cc1</smiles><smiles>CCOc1ccccc1F</smiles><smiles>CCOc1c(F)cccc1F</smiles><smiles>CCOc1ccc(C)cc1</smiles><smiles>CCOc1ccccc1C</smiles><smiles>CCOc1cccc(C)c1</smiles><smiles>CCOc1ccc(N)cc1</smiles><smiles>CCOc1cccc2ccccc12</smiles><smiles>CCOc1ccc2ccccc2c1</smiles>
$11,74 \%$<smiles>CCOc1ccc(C#N)cc1</smiles><smiles>COc1ccc([N+](=O)[O-])cc1</smiles><smiles>O=C1CCCc2ccccc21</smiles>

$16,76 \%^{* * *}$

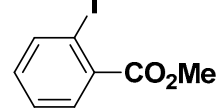

$17,89 \% * * * *$

Figure 2. Alkylation of aryl alcohols by boron trifluoride etherates. ${ }^{*}$ Reflux overnight. ${ }^{* *}$ Reflux of 4-phenylbutyric acid with boron trifluoride methyl etherate. ${ }^{* *}$ Reflux of the aryl alcohol in boron trifluoride methyl etherate. ${ }^{* * * *}$ Reflux of o-iodobenzoic acid with boron trifluoride methyl etherate.

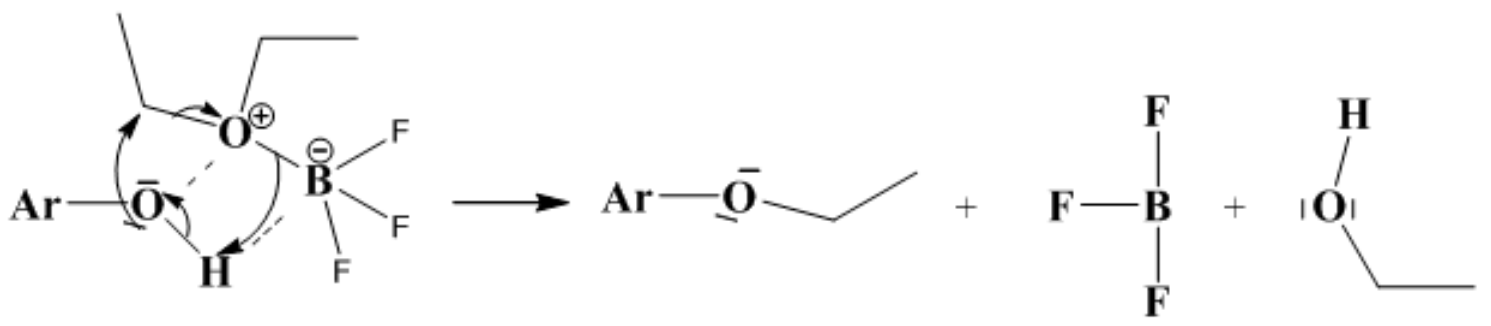

Scheme 1. Proposed alkylation mechanism.

A comparison between reported results of the synthesis of selected aryl ethyl ethers with the results obtained in the present work is shown in Table 1. The merit of this method lies in its novelty and simplicity, consisting merely in refluxing the substrate in BTE. 
Table 1. Comparison of the results obtained for the synthesis of aryl ethyl ethers with those recently reported in the literature.

\begin{tabular}{|c|c|c|c|c|}
\hline Product & This Work (\%) & Literature Conditions & Literature Yield (\%) & Reference \\
\hline Phenetole 1 & 84.8 & $\begin{array}{l}\text { Phenol/EtONa/diethyl carbonate, } 137 \\
{ }^{\circ} \mathrm{C} / 42 \mathrm{~h} .\end{array}$ & 71 & [4] \\
\hline p-Chloroethoxybenzene 2 & 95 & $\begin{array}{c}p \text {-Chlorophenol } / \mathrm{K}_{2} \mathrm{CO}_{3} / \mathrm{EtBr} / \text { Acetone, } \\
\text { reflux, } 3 \mathrm{~h} .\end{array}$ & 89 & [20] \\
\hline$p$-Ethoxytoluene 11 & 88 & $\begin{array}{l}p \text {-Iodotoluene/NMP, } 170{ }^{\circ} \mathrm{C} / \mathrm{Cu}_{2} \mathrm{O} \\
(5 \%), \mathrm{CsCO}_{3}(2 \mathrm{eqv}), \mathrm{MW}, 30 \mathrm{~min} .\end{array}$ & 77 & [21] \\
\hline p-Nitroethoxybenzene 4 & 72 & $\begin{array}{l}p \text {-Fluoronitrobenzene/EtOH, Triton } \\
\mathrm{B} / 50^{\circ} \mathrm{C}, 20 \mathrm{~h} .\end{array}$ & 93 & [22] \\
\hline$\beta$-Ethoxynaphthalene 18 & 85 & $\begin{array}{c}\text { 2-naphthol/EtBr/NaOH } \\
40 \% / \mathrm{TBAB} / \mathrm{Toluene}, 70^{\circ} \mathrm{C}, 4 \mathrm{~h} .\end{array}$ & 98.1 & [23] \\
\hline
\end{tabular}

Similar reactions using boron trifluoride methyl etherate with either carboxylic acids [1] and phenols produced similar results (see compounds 14, 15, and 17), with the only notable exception of the reaction with 4-phenylbutyric acid, which led to the formation of $\alpha$-tetralone, the product of the intramolecular Friedel-Crafts acylation in $76 \%$ yield. These results show that boron trifluoride methyl etherate is a stronger Lewis acid than BTE.

\section{Materials and Methods}

\section{General Information}

Solvents (n-hexane, ethyl acetate) were purchased from Shalom Laboratory Supplies cc; TLC plates (silica 60) were obtained from DLD Scientific SA cc; boron trifluoride etherate and boron trifluoride dimethyl etherate were procured from Merck SA (Pty) Ltd; anhydrous sodium sulfate was obtained from Prestige Laboratory Supplies and anhydrous calcium chloride was purchased from United Scientific SA cc. All the above chemicals were used without further purification. Products were characterized by ${ }^{1} \mathrm{H}$ - and ${ }^{13} \mathrm{C}-\mathrm{NMR}$ spectroscopy using a Varian spectrometer at 400 and $100 \mathrm{MHz}$ for proton and carbon-13, respectively. Spectra were recorded from $\mathrm{CDCl}_{3}$ solutions employing TMS as an internal reference. All spectra are reported as $\delta(\mathrm{ppm})$ values. Please find the NMR spectra of compounds in Supplementary Materials.

General procedure (compound 1 [4,11]): In a $50 \mathrm{~mL}$ round-bottomed flask equipped with a magnetic stirring bar, a reflux condenser and a calcium chloride drying tube were placed phenol $(1 \mathrm{~g}, 10.6 \mathrm{mmol})$ and boron trifluoride etherate $(10 \mathrm{~mL}, 77 \mathrm{mmol})$. The reaction mixture was stirred and heated to $120^{\circ} \mathrm{C}$ for four hours during which the progress of the reaction was monitored by thin layer chromatography (n-hexane/ethyl acetate 7:1). The cooled reaction mixture was carefully diluted with water $(20 \mathrm{~mL})$ and extracted with $\mathrm{n}$-hexane. The aqueous phase was further extracted with $\mathrm{n}$-hexane $(20 \mathrm{~mL})$. The combined organic extract was washed with water $(10 \mathrm{~mL})$ followed by a saturated solution of $\mathrm{NaHCO}_{3}(10 \mathrm{~mL})$ until complete cessation of effervescence. The organic layer was separated and dried over anhydrous $\mathrm{Na}_{2} \mathrm{SO}_{4}$ and concentrated in vacuo affording a clear liquid, $1.23 \mathrm{~g}(94.9 \%)$. Column chromatography on silica gel (n-hexane/ethyl acetate 7:1) gave $1.1 \mathrm{~g}(84.8 \%)$ of a pleasant-smelling oil. $\delta_{\mathrm{H}}\left(400 \mathrm{MHz}, \mathrm{CDCl}_{3}\right):{ }^{1} \mathrm{H} \mathrm{NMR}(400 \mathrm{MHz}$, Chloroform-d) $\delta 7.23-7.09(\mathrm{~m}, 2 \mathrm{H})$, 6.89-6.76 (m, 3H), $3.93(\mathrm{q}, \mathrm{J}=7.0 \mathrm{~Hz}, 2 \mathrm{H}), 1.32(\mathrm{t}, \mathrm{J}=7.0,3 \mathrm{H}) ;{ }^{13} \mathrm{C}$ NMR (101 MHz, Chloroform-d) $\delta$ $158.93,129.43,120.53,114.47,63.27,14.86$.

Procedure for the preparation of $\alpha$-tetralone (compound 16 [24]): A $50 \mathrm{~mL}$ round-bottomed flask equipped with a magnetic stirring bar, a reflux condenser and a calcium chloride drying tube was charged 4-phenylbutyric acid $(1 \mathrm{~g}, 6.1 \mathrm{mmol})$ and boron trifluoride dimethyl etherate $(10 \mathrm{~mL}$, $113 \mathrm{mmol})$. The reaction mixture was stirred and heated reflux for four hours during after which the thin layer chromatography analysis (n-hexane/ethyl acetate 5:1) showed that the reaction was complete. The cooled reaction mixture was carefully diluted with water $(20 \mathrm{~mL})$ and extracted with $\mathrm{n}$-hexane. The aqueous phase was further extracted with n-hexane $(20 \mathrm{~mL})$. The combined organic extract was washed with water $(10 \mathrm{~mL})$ followed by a saturated solution of $\mathrm{NaHCO}_{3}(10 \mathrm{~mL})$ until complete 
cessation of effervescence. The organic layer was separated and dried over anhydrous $\mathrm{Na}_{2} \mathrm{SO}_{4}$ and concentrated in vacuo affording a clear liquid which was purified by column chromatography on silica gel (n-hexane/ethyl acetate 5:1) to give clear liquid (0.622 g, 76\%). ${ }^{1} \mathrm{H} \mathrm{NMR} \mathrm{(400} \mathrm{MHz,} \mathrm{Chloroform-} d$ ) $\delta$ $8.00(\mathrm{~d}, J=7.7 \mathrm{~Hz}, 1 \mathrm{H}), 7.47-7.39(\mathrm{t}, J=7.7 \mathrm{~Hz}, 1 \mathrm{H}), 7.27(\mathrm{t}, J=7.7 \mathrm{~Hz}, 1 \mathrm{H}), 7.22(\mathrm{~d}, J=7.7 \mathrm{~Hz}, 1 \mathrm{H}), 2.93$ $(\mathrm{t}, J=6.0 \mathrm{~Hz}, 2 \mathrm{H}), 2.62(\mathrm{t}, J=6.0 \mathrm{~Hz}, 2 \mathrm{H}), 2.10(\mathrm{p}, J=6.4 \mathrm{~Hz}, 2 \mathrm{H}) ;{ }^{13} \mathrm{C}$ NMR $(101 \mathrm{MHz}$, Chloroform- $d) \delta$ $198.22,144.36,133.26,132.47,128.65,126.99,126.47,39.02,29.56,23.15$.

p-Ethoxychlorobenzene 2 [20]: clear liquid, $1.13 \mathrm{~g}(93 \%) ;{ }^{1} \mathrm{H}$ NMR $(400 \mathrm{MHz}$, Chloroform- $d) \delta 7.17-7.08$ $(\mathrm{m}, 2 \mathrm{H}), 6.75-6.67(\mathrm{~m}, 2 \mathrm{H}), 3.88(\mathrm{q}, \mathrm{J}=7.0 \mathrm{~Hz}, 2 \mathrm{H}), 1.30(\mathrm{t}, J=7.0 \mathrm{~Hz}, 3 \mathrm{H}) ;{ }^{13} \mathrm{C} \mathrm{NMR}(101 \mathrm{MHz}$, Chloroform-d) $\delta 157.55,129.24,125.31,115.72,63.71,14.72$.

Ethoxy-2,4-dichlorobenzene 3 [25]: clear liquid, $0.91 \mathrm{~g}(78 \%) ;{ }^{1} \mathrm{H}$ NMR (400 MHz, Chloroform- $\left.d\right) \delta 7.27$ (d, $J=2.6 \mathrm{~Hz}, 1 \mathrm{H}), 7.07(\mathrm{dd}, J=8.8,2.6 \mathrm{~Hz}, 1 \mathrm{H}), 6.73(\mathrm{~d}, J=8.8 \mathrm{~Hz}, 1 \mathrm{H}), 3.98(\mathrm{q}, J=7.0 \mathrm{~Hz}, 2 \mathrm{H}), 1.37(\mathrm{t}, J=$ 7.0 Hz, 3H); ${ }^{13} \mathrm{C}$ NMR (101 MHz, Chloroform-d) $\delta 153.28,129.89,127.46,125.42,123.59,113.96,65.00$, 14.59 .

p-Ethoxynitrobenzene 4 [10]: pale yellow solid, $0.87 \mathrm{~g}(72 \%) ;{ }^{1} \mathrm{H}$ NMR (400 MHz, Chloroform- $d$ ) $\delta 8.18$ $(\mathrm{d}, J=9.1 \mathrm{~Hz}, 2 \mathrm{H}), 6.93(\mathrm{~d}, J=9.1 \mathrm{~Hz}, 2 \mathrm{H}), 4.12(\mathrm{q}, J=6.9 \mathrm{~Hz}, 2 \mathrm{H}), 1.45(\mathrm{t}, J=7.0 \mathrm{~Hz}, 3 \mathrm{H}) .{ }^{13} \mathrm{C} \mathrm{NMR}$ (101 MHz, Chloroform-d) $\delta$ 164.03, 125.92, 114.35, 64.40, 14.55.

o-Ethoxyfluorobenzene 5 [26]: clear liquid, $0.74 \mathrm{~g} \mathrm{(59 \% );}{ }^{1} \mathrm{H}$ NMR $(400 \mathrm{MHz}$, Chloroform) $\delta 6.86-7.11$ (m, $4 \mathrm{H}), 4.11(\mathrm{q}, J=7.0 \mathrm{~Hz}, 2 \mathrm{H}), 1.46(\mathrm{t}, J=7.0 \mathrm{~Hz}, 3 \mathrm{H}),{ }^{13} \mathrm{C}$ NMR $(101 \mathrm{MHz}$, Chloroform- $d) \delta 152.75\left(\mathrm{~d}, J_{C-F}\right.$ $=245.25), 146.95\left(\mathrm{~d}, \mathrm{~J}_{\mathrm{C}-\mathrm{F}}=10.12\right), 124.20\left(\mathrm{~d}, J_{\mathrm{C}-\mathrm{F}}=3.89\right), 120.85\left(\mathrm{~d}, J_{\mathrm{C}-\mathrm{F}}=7.00\right), 116.08\left(\mathrm{~d}, J_{\mathrm{C}-\mathrm{F}}=17.91\right)$, $114.84\left(\mathrm{~d}, J_{\mathrm{C}-F}=1.55\right), 64.80,14.75$.

Ethoxy-2,6-difluorobenzene 6 [27]: clear liquid, $0.63 \mathrm{~g}$ (51.7\%); ${ }^{1} \mathrm{H}$ NMR (400 MHz, Chloroform-d) $\delta$ 6.98-6.73 (m, 3H), $4.18(\mathrm{qm}, J=7.0,2 \mathrm{H}), 1.37(\mathrm{t}, J=7.0,3 \mathrm{H}) ;{ }^{13} \mathrm{C}$ NMR (101 MHz, Chloroform-d) $\delta$ $156.41\left(\mathrm{dd}, J_{C-F}=248.34,5.45\right), 135.44\left(\mathrm{t}, J_{C-F}=14.41\right), 122.60\left(\mathrm{t}, J_{C-F}=9.34\right), 112.04\left(\mathrm{dd}, J_{C-F}=16.35\right.$, 6.23), 64.80, 14.75 .

p-Ethoxytoluene 7 [21]: clear liquid, $1.11 \mathrm{~g}(88 \%) ;{ }^{1} \mathrm{H}$ NMR $(400 \mathrm{MHz}$, Chloroform- $d$ ) $\delta$ 7.02-6.95 (m, $2 \mathrm{H}), 6.75-6.67(\mathrm{~m}, 2 \mathrm{H}), 3.91(\mathrm{q}, \mathrm{J}=7.0,2 \mathrm{H}), 2.19(\mathrm{~s}, 3 \mathrm{H}), 1.31(\mathrm{t}, J=7.0,3 \mathrm{H}) ;{ }^{13} \mathrm{C}$ NMR $(101 \mathrm{MHz}$, Chloroform-d) $\delta 156.76,129.82,129.64,114.28,63.36,20.41,14.85$.

o-Ethoxytoluene 8 [21]: clear liquid, $\left.0.67 \mathrm{~g}(53 \%) ;{ }^{1} \mathrm{H} \mathrm{NMR} \mathrm{(400} \mathrm{MHz,} \mathrm{Chloroform-} d\right) \delta 7.56-7.46(\mathrm{~m}, 2 \mathrm{H})$, 7.26-7.13 (m, 2H), $4.39(\mathrm{q}, J=7.0 \mathrm{~Hz}, 2 \mathrm{H}), 2.61(\mathrm{~s}, 3 \mathrm{H}), 1.79(\mathrm{t}, J=7.0 \mathrm{~Hz}, 3 \mathrm{H}) ;{ }^{13} \mathrm{C}$ NMR $(101 \mathrm{MHz}$, Chloroform- $d$ ) $\delta 159.02,139.40,129.18,121.38,115.40,111.38,63.23,21.52,14.90$.

$m$-Ethoxytoluene 9 [4]: clear liquid, $1.13 \mathrm{~g}(90 \%) ;{ }^{1} \mathrm{H}$ NMR (400 MHz, Chloroform- $\left.d\right) \delta 7.19(\mathrm{t}, J=8.1 \mathrm{~Hz}$, $1 \mathrm{H}), 6.56-6.46(\mathrm{~m}, 3 \mathrm{H}), 4.03(\mathrm{q}, J=7.0 \mathrm{~Hz}, 2 \mathrm{H}), 3.80(\mathrm{~s}, 3 \mathrm{H}), 1.43(\mathrm{t}, J=7.0 \mathrm{~Hz}, 3 \mathrm{H}) ;{ }^{13} \mathrm{C} \mathrm{NMR}(101 \mathrm{MHz}$, Chloroform- $d$ ) $\delta 159.02,139.40,129.18,121.38,115.40,111.38,63.23,21.52,14.90$.

p-Ethoxyaniline 10 [28]: light yellow liquid, $0.21 \mathrm{~g}(17 \%) ;{ }^{1} \mathrm{H}$ NMR (400 MHz, Chloroform) $\delta 6.69-6.62$ $(\mathrm{m}, 2 \mathrm{H}), 6.55(\mathrm{~d}, \mathrm{~J}=8.7 \mathrm{~Hz}, 2 \mathrm{H}), 3.87(\mathrm{q}, \mathrm{J}=7.0 \mathrm{~Hz}, 2 \mathrm{H}), 3.26(\mathrm{bs}, 2 \mathrm{H}), 1.29(\mathrm{t}, J=7.0 \mathrm{~Hz}, 3 \mathrm{H}) ;{ }^{13} \mathrm{C} \mathrm{NMR}$ (101 MHz, Chloroform-d) $\delta$ 152.13, 139.79, 116.44, 115.67, 64.04, 14.95.

$\alpha$-Ethoxynaphthalene 11 [28]: clear liquid, $0.88 \mathrm{~g}(74 \%){ }^{1} \mathrm{H}$ NMR $(400 \mathrm{MHz}$, Chloroform- $d) \delta$ 7.64-7.82 $(\mathrm{m}, 3 \mathrm{H}), 7.47-7.51(\mathrm{~m}, 1 \mathrm{H}), 7.36-7.40(\mathrm{~m}, 1 \mathrm{H}), 7.16-7.23(\mathrm{~m}, 2 \mathrm{H}), 4.26(\mathrm{q}, J=6.85 \mathrm{~Hz}, 2 \mathrm{H}), 1.64(\mathrm{t}, \mathrm{J}=$ $6.85 \mathrm{~Hz}, 3 \mathrm{H}) ;{ }^{13} \mathrm{C}$ NMR (101 MHz, Chloroform-d) $\delta 156.9,134.59,129.29,128.87,127.59,126.67,126.23$, $123.44,118.96,106.51,63.35,14.75$.

$\beta$-Ethoxynaphthalene 12 [4,23]: clear liquid, $1.01 \mathrm{~g}(85 \%) ;{ }^{1} \mathrm{H}$ NMR (400 MHz, Chloroform- $d$ ) $\delta 8.47-8.50$ $(\mathrm{m}, 1 \mathrm{H}), 7.91-7.93(\mathrm{~m}, 1 \mathrm{H}), 7.46-7.63(\mathrm{~m}, 4 \mathrm{H}), 6.87(\mathrm{~d}, \mathrm{~J}=7.83 \mathrm{~Hz}, 1 \mathrm{H}), 4.26(\mathrm{q}, J=7.0 \mathrm{~Hz}, 2 \mathrm{H}), 1.64$ $(\mathrm{t}, \mathrm{J}=7.0 \mathrm{~Hz}, 3 \mathrm{H}) ;{ }^{13} \mathrm{C}$ NMR $(101 \mathrm{MHz}$, Chloroform- $d$ ) $\delta 154.70,134.47,127.35,126.23,125.85,125.70$, $124.96,122.06,119.93,104.51,63.55,14.74$. 
p-Ethoxybenzonitrile 13 [29]: white solid, $0.25 \mathrm{~g}(20 \%) ;{ }^{1} \mathrm{H}$ NMR (400 MHz, Chloroform) $\delta 7.54-7.46$ $(\mathrm{m}, 2 \mathrm{H}), 6.90-6.82(\mathrm{~m}, 2 \mathrm{H}), 4.01(\mathrm{q}, \mathrm{J}=7.0 \mathrm{~Hz}, 2 \mathrm{H}), 1.37(\mathrm{t}, J=7.0 \mathrm{~Hz}, 3 \mathrm{H}) ;{ }^{13} \mathrm{C} \mathrm{NMR}(101 \mathrm{MHz}$, Chloroform- $d$ ) $\delta 162.23,133.94,119.27,115.13,103.69,63.90,14.53$.

p-Chloroanisole 14 [30]: colourless liquid, $0.82 \mathrm{~g}(75 \%){ }^{1} \mathrm{H}$ NMR (400 MHz, Chloroform) $\delta 7.24$ (d, $J=$ $8.7 \mathrm{~Hz}, 2 \mathrm{H}), 6.83(\mathrm{~d}, J=8.7 \mathrm{~Hz}, 2 \mathrm{H}), 3.78(\mathrm{~s}, 3 \mathrm{H}) .{ }^{13} \mathrm{C}$ NMR $(101 \mathrm{MHz}$, Chloroform- $d$ ) $\delta$ 158.10, 129.23, $125.43,115.06,55.40$.

p-Nitroanisole 15 [31]: white solid, $0.88 \mathrm{~g}(80 \%),{ }^{1} \mathrm{H}$ NMR $(400 \mathrm{MHz}$, Chloroform) $\delta 8.17$ (d, J = $9.2 \mathrm{~Hz}$, 2H), $6.93(\mathrm{~d}, J=9.2 \mathrm{~Hz}, 2 \mathrm{H}), 3.89(\mathrm{~s}, 3 \mathrm{H}) .{ }^{13} \mathrm{C}$ NMR (101 MHz, Chloroform-d) $\delta 164.52,141.51,125.79$, $113.84,55.78$.

Methyl o-iodobenzoate 17 [32]; colorless liquid, $0.9441 \mathrm{~g}$ ( $89 \%$ yield. ${ }^{1} \mathrm{H}$ NMR (400 MHz, Chloroform) $\delta$ $7.95(\mathrm{~d}, J=7.83 \mathrm{~Hz}, 1 \mathrm{H}), 7.76(\mathrm{~d}, J=7.44 \mathrm{~Hz}, 1 \mathrm{H}), 7.36(\mathrm{t}, J=7.63 \mathrm{~Hz}, 1 \mathrm{H}), 3.90-7.11(\mathrm{t}, J=7.44 \mathrm{~Hz}, 1 \mathrm{H})$. ${ }^{13} \mathrm{C}$ NMR (101 MHz, Chloroform-d) $\delta 166.80,141.17,134.88,132.56,130.82,127.79,94.00,55.20$.

\section{Conclusions}

This reaction compares more favorably in terms of cost and simplicity to other potential processes, especially those listed in Table 1 above.

While the high cost of BTE may be a hindrance to a wide scale industrial application of this ethylation protocol compared to the traditional Williamson ether synthesis, it may be of use in the pharmaceutical and fine chemical industries, where batch reactions are widely in operation and emphasis is on small production volumes.

Supplementary Materials: The NMR spectra of compounds obtained in this study are available online.

Author Contributions: Conceptualization, W.M.; methodology, W.M. and N.D.J.; experiments, Y.M., N.I.M., E.M. and S.M.; writing-original draft preparation, W.M.; writing-review and editing, N.D.J.

Funding: This research was funded by Walter Sisulu University Directorate of Research Development and Innovation.

Conflicts of Interest: The authors declare no conflict of interest.

\section{References}

1. Jumbam, N.D.; Maganga, Y.; Masamba, W.; Mbunye, N.I.; Mgoqi, E.; Mtwa, S. Unprecedented alkylation of carboxylic acids by boron trifluoride etherate. Bull. Chem. Soc. Ethiopia 2018, 32, 387-392. [CrossRef]

2. Sutter, M.; Lafon, R.; Raoul, Y.; Métay, E.; Lemaire, M. Heterogeneous Palladium-catalyzed synthesis of aromatic ethers by solvent-free dehydrogenative aromatization: Mechanism, scope, and limitations under aerobic and non-aerobic conditions. Eur. J. Org. Chem. 2013, 2013, 5902-5916. [CrossRef]

3. Mirafzal, G.A.; Summer, J.M. Microwave irradiation reactions: Synthesis of analgesic drugs. J. Chem. Educ. 2000, 77, 356-357. [CrossRef]

4. Weidlich, T.; Pokorný, M.; Padělková, Z.; Růžička, A. Aryl ethyl ethers prepared by ethylation using diethyl carbonate. Green Chem. Lett. Rev. 2007, 1, 53-59. [CrossRef]

5. Kim, J.W.; Kim, D.J.; Han, J.U.; Kang, M.; Kim, J.M.; Yie, J.E. Preparation and characterization of zeolite catalysts for etherification reaction. Catal. Today 2003, 87, 195-203. [CrossRef]

6. Shindo, K.; Tachibana, A.; Tanaka, A.; Toba, S.; Yuki, E.; Ozaki, T.; Kumano, T.; Nishiyama, M.; Misawa, N.; Kuzuyama, T. Production of novel antioxidative prenyl naphthalen-ols by combinational bioconversion with dioxygenase PhnA1A2A3A4 and prenyltransferase NphB or SCO7190. Biosci. Biotechnol. Biochem. 2011, 75, 505-510. [CrossRef]

7. Malykhin, E.V.; Shteingarts, V.D. Alkoxylation of 4-chloronitrobenzene with aliphatic alcohols and glycols in the presence of NaOH. Russ. J. Appl. Chem. 2012, 85, 1232-1238. [CrossRef]

8. Sun, H.; Sun, Y.; Tian, X.; Zhao, Y.; Qi, X. Nanosized ferric hydroxide catalyzed C-O cross-coupling of phenol and halides to generate phenoxy ether. Asian J. Chem. 2013, 25, 6189-6191. [CrossRef]

9. Smith, R.G.; Vanterpool, A.; Kulak, H.J. Dimethyl sulfoxide as a solvent in the Williamson ether synthesis. Can. J. Chem. 1969, 47, 2015-2020. [CrossRef] 
10. Xionga, W.; Dinga, Q.; Chena, J.; Dinga, J.; Wua, H. Solvent-free synthesis of aryl ethers promoted by tetrabutylammonium fluoride. J. Chem. Res. 2010, 34, 395-398. [CrossRef]

11. Keegstra, M.A.; Peters, T.H.; Brandsma, L. Copper (I) halide catalysed synthesis of alkyl aryl and alkyl heteroaryl ethers. Tetrahedron 1992, 48, 3633-3652. [CrossRef]

12. Milton, E.J.; Fuentes, J.A.; Clarke, M.L. Palladium-catalysed synthesis of aryl-alkyl ethers using alkoxysilanes as nucleophiles. Org. Biomol. Chem. 2009, 7, 2645-2648. [CrossRef] [PubMed]

13. Paul, S.; Gupta, M. Zinc-catalyzed Williamson ether synthesis in the absence of base. Tetrahedron Lett. 2004, 45, 8825-8829. [CrossRef]

14. Manhas, M.S.; Hoffman, W.; Lal, B.; Bose, A.K. Steroids. Part X. A convenient synthesis of alkyl aryl ethers. J. Chem. Soc. Perk. Trans. 1 1975, 5, 461-463. [CrossRef]

15. Guerrero, L.R.; Rivero, I.A. 1,2-Dimethylimidazole (DMI) and microwaves in the alkylation of carboxylic acids and phenols with dimethyl and diethyl carbonates. Arkivoc 2008, 11, 295-306.

16. Kreye, O.; Over, L.C.; Nitsche, T.; Lange, R.Z.; Meier, M.A.R. Organic carbonates: Sustainable and environmentally-friendly ethylation, allylation, and benzylation reagents. Tetrahedron 2015, 71, 293-300. [CrossRef]

17. Heller, S.T.; Sarpong, R. On the reactivity of imidazole carbamates and ureas and their use as esterification and amidation reagents. Tetrahedron 2011, 67, 8851-8859. [CrossRef]

18. Kobayashi, A.; Konishi, G.-I. Rapid synthesis of phenolic resins by microwave-assisted self-condensation of hydroxybenzyl alcohol derivatives. Polym. J. 2008, 40, 590-591. [CrossRef]

19. Posner, G.H.; Shulman-Roskes, E.M.; Oh, C.H.; Carry, J.-C.; Green, J.V.; Clark, A.B.; Dai, H.; Anjeh, T.E.N. BF3.OEt2 promotes fast, mild, clean and regioselective dehydration of tertiary alcohols. Tetrahedron Lett. 1991, 32, 6489-6492. [CrossRef]

20. Bouider, N.; Fhayli, W.; Ghandour, Z.; Boyer, M.; Harrouche, K.; Florence, X.; Pirotte, B.; Lebrun, P.; Faury, G.; Khelili, S. Design and synthesis of new potassium channel activators derived from the ring opening of diazoxide: Study of their vasodilatory effect, stimulation of elastin synthesis and inhibitory effect on insulin release. Bioorg. Med. Chem. 2015, 23, 1735-1746. [CrossRef]

21. Mehmood, A.; Devine, W.G.; Leadbeater, N.E. Development of methodologies for copper-catalyzed C-O bond formation and direct cyanation of aryl iodides. Top. Catal. 2010, 53, 1073-1080. [CrossRef]

22. Meshram, H.M.; Goud, P.R.; Reddy, B.C.; Kumar, D.A. Triton B-Mediated Efficient and Convenient Alkoxylation of Activated Aryl and Heteroaryl Halides. Synth. Commun. 2010, 40, 2122-2129. [CrossRef]

23. Mathew, S.H.; Haridas, K.R. Synthesis and properties of N,N, $N^{\prime}$-tris-(2-ethoxy-naphthalenen-1-yl)-N,N,N' triphenylbenzene 1, 3, 5-triamine for dye sensitized solar cell. Bull. Mater. Sci. 2012, 35, 123-127. [CrossRef]

24. Tang, G.; Gong, Z.; Han, W.; Sun, X. Visible light mediated aerobic photocatalytic activation of CH bond by riboflavin tetraacetate and N-hydroxysuccinimide. Tetrahedron Lett. 2018, 59, 658-662. [CrossRef]

25. Foglia, T.A.; Swern, D. Reaction of N,N-dichlorourethan with ethers. A novel cleavage-chlorination reaction. Tetrahedron Lett. 1967, 8, 3963-3967. [CrossRef]

26. Dubbaka, S.R.; Narreddula, V.R.; Gadde, S.; Mathew, T. Silver-mediated fluorination of potassium aryltrifluoroborates with Selectfluorß. Tetrahedron 2014, 70, 9676-9681. [CrossRef]

27. Yunlong, J.; Yuan, Y.; Jin, D. Method for preparing difluoro alkyl ether benzene. U.S. Patent No. CN101003468 (A), 25 July 2007.

28. Kumar, A.; Kumar, P.; Paul, S.; Jain, S.L. Visible light assisted reduction of nitrobenzenes using Fe(bpy)3+2/rGO nanocomposite as photocatalyst. Appl. Surf. Sci. 2016, 386, 103-114. [CrossRef]

29. Xu, B.; Jiang, Q.; Zhao, A.; Jia, J.; Liu, Q.; Luo, W.; Guo, C. Copper-catalyzed aerobic conversion of the C=O bond of ketones to a $\mathrm{C} \equiv \mathrm{N}$ bond using ammonium salts as the nitrogen source. Chem. Commun. 2015, 51, 11264-11267. [CrossRef]

30. Lui, M.Y.; Yuen, A.K.L.; Masters, A.F.; Maschmeyer, T. Masked N-heterocyclic carbene-catalyzed alkylation of phenols with organic carbonates. ChemSusChem 2016, 9, 2312-2316. [CrossRef]

31. Maleki, A.; Aghaei, M.; Paydar, R. Highly efficient protocol for the aromatic compounds nitration catalyzed by magnetically recyclable core/shell nanocomposite. J. Iran. Chem. Soc. 2017, 14, 485-490. [CrossRef]

32. Curti, F.; Tiecco, M.; Pirovano, V.; Germani, R.; Caselli, A.; Rossi, E.; Abbiati, G. p-TSA-Based DESs as "Active Green Solvents" for Microwave Enhanced Cyclization of 2-Alkynyl-(hetero)-arylcarboxylates: An alternative access to 6-substituted 3,4-fused 2-pyranones. Eur. J. Org. Chem. 2019, 2019, 1904-1914. [CrossRef] 
Sample Availability: Samples of the compounds 1-17 are available from the authors.

(C) 2019 by the authors. Licensee MDPI, Basel, Switzerland. This article is an open access article distributed under the terms and conditions of the Creative Commons Attribution (CC BY) license (http://creativecommons.org/licenses/by/4.0/). 